\title{
Cluster observations of ELF/VLF signals generated by modulated heating of the lower ionosphere with the HAARP HF transmitter
}

\author{
M. Platino ${ }^{1}$, U. S. Inan ${ }^{1}$, T. F. Bell ${ }^{1}$, J. Pickett ${ }^{2}$, E. J. Kennedy ${ }^{3}$, J. G. Trotignon ${ }^{4}$, J. L. Rauch ${ }^{4}$, and P. Canu ${ }^{5}$ \\ ${ }^{1}$ Space, Telecommunications and Radioscience (STAR) Laboratory, Stanford University, Stanford, CA 94305, USA \\ ${ }^{2}$ Dept. of Physics and Astronomy, University of Iowa, Iowa City, IA 52242, USA \\ ${ }^{3}$ Naval Research Laboratory, 4555 Overlook Ave. SW, Washington D.C. 20375, USA \\ ${ }^{4}$ Laboratoire de Physique et Chimie de l'Environnement, Centre National de la Recherche Scientifique, 3 A avenue de la \\ Recherche Scientifique, F-45071 Orléans cedex 02, France \\ ${ }^{5}$ Centre d'Etudes des Environnements Terrestres et Planétaires, Centre National de la Recherche Scientifique, Université \\ Versailles Saint-Quentin-en-Yvelines, 10 avenue de l'Europe, F-78140, Vélizy Villacoublay, France
}

Received: 9 October 2003 - Revised: 29 April 2004 - Accepted: 18 May 2004 - Published: 14 July 2004

Part of Special Issue "Spatio-temporal analysis and multipoint measurements in space"

\begin{abstract}
It is now well known that amplitude modulated $\mathrm{HF}$ transmissions into the ionosphere can be used to generate ELF/VLF signals using the so-called "electrojet antenna". Although most observations of the generated ELF/VLF signals have been made on the ground, several low and highaltitude satellite observations have also been reported (James et al., 1990). One of the important unknowns in the physics of ELF/VLF wave generation by ionospheric heating is the volume of the magnetosphere illuminated by the ELF/VLF waves. In an attempt to investigate this question further, ground-satellite conjunction experiments have recently been conducted using the four Cluster satellites and the HF heater of the High-Frequency Active Auroral Research Program (HAARP) facility in Gakona, Alaska. Being located on largely closed field lines at $L \approx 4.9$, HAARP is currently also being used for ground-to-ground type of ELF/VLF waveinjection experiments, and will be increasingly used for this purpose as it is now being upgraded for higher power operation. In this paper, we describe the HAARP installation and present recent results of the HAARP-Cluster experiments. We give an overview of the detected ELF/VLF signals at Cluster, and a possible explanation of the spectral signature detected, as well as the determination of the location of the point of injection of the HAARP ELF/VLF signals into the magnetosphere using ray tracing.
\end{abstract}

Key words. Magnetospheric physics (magnetosphereionosphere interactions) - Radio science (radio wave propagation) - Space plasma physics (active perturbation experiments)

Correspondence to: M. Platino

(platinom@stanford.edu)

\section{Introduction}

To radiate radio waves efficiently requires antennas whose dimensions are of the order of the radio wavelength being emitted. At very low frequencies (VLF), where wavelengths range from $10 \mathrm{~km}$ to $1000 \mathrm{~km}$, it is therefore not surprising that attempts have been made to exploit large-scale geophysical current systems as radiating elements. Stubbe and Kopka (1977) suggested using a powerful High Frequency (HF) heater, modulated at an audio frequency, to change the D-region conductivity in the auroral zone in the vicinity of the auroral electrojet and thus produce a huge oscillating VLF dipole source within the ionosphere. The mechanism of generation of the VLF waves using a powerful HF ionospheric heater involves the modification of the electron temperature in the $\mathrm{D}$ - and lower E-regions of the ionosphere. The modified electron temperature leads to changes in the Pedersen and Hall conductivities and thus to changes in ionospheric current density. If the HF carrier is modulated in a periodic way (either frequency or amplitude), the current density is periodically changed. Under such circumstances, the heated ionospheric area with its immediate surroundings becomes a huge antenna radiating at a frequency corresponding to the modulation frequency of the HF carrier.

In a number of experiments it has been demonstrated that a carrier HF wave, modulated in amplitude by a VLF wave, can have a higher Effective Radiated Power when the HF signal is in the $\mathrm{X}$ mode rather than the $\mathrm{O}$ mode. A significant body of data from ground VLF observations at different heating facilities has been used to establish the characteristics of downward radiation from the auroral radiator (Kapustin et al., 1977; Stubbe et al., 1981, 1982; Ferraro et al., 1982; 
(a)

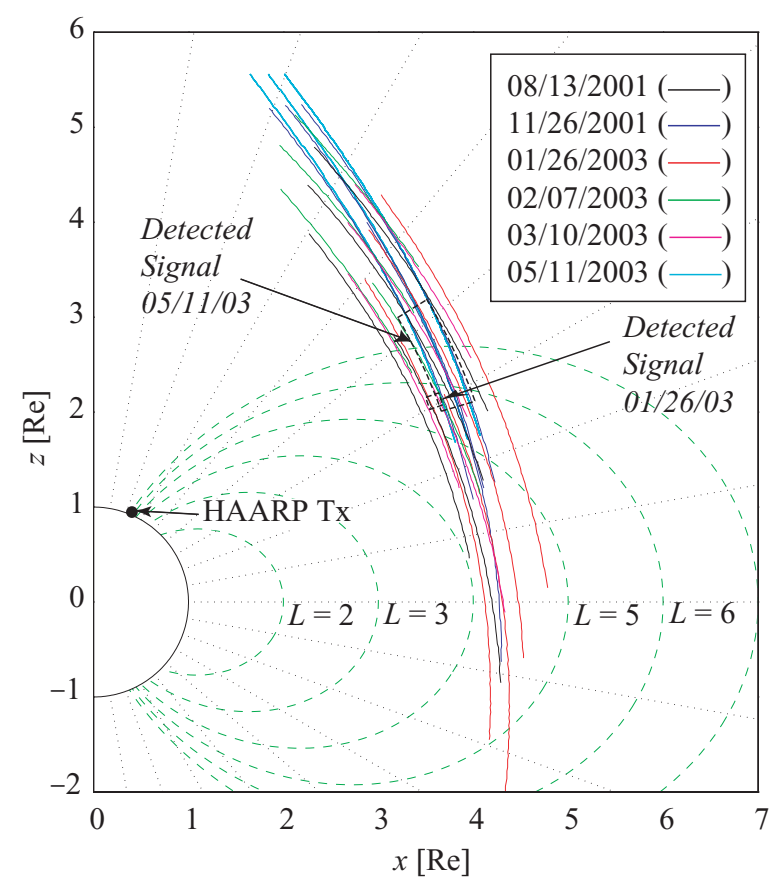

(b)

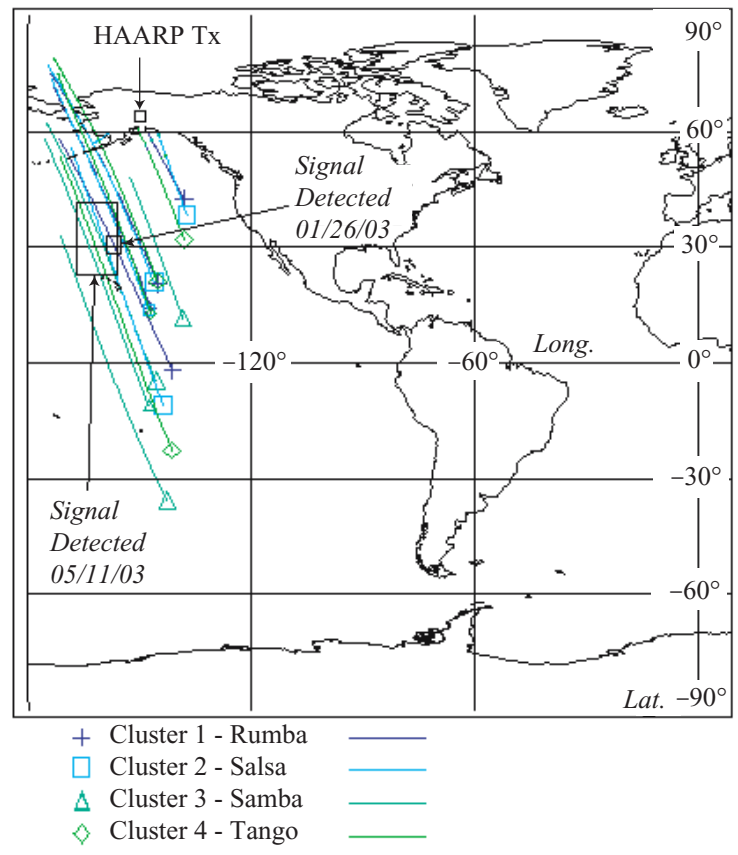

Fig. 1. Trajectories of the Cluster spacecraft during the dates when the HAARP-Cluster experiment was performed. (a) Projection of the spacecraft trajectories upon a meridional plane. Each color corresponds to a different day, as highlighted in the legend box. Magnetic coordinates are based on the centered dipole model. (b) Geographic projection of the spacecraft trajectories. Each color corresponds to a different spacecraft, as highlighted in the legend box. The dashed line rectangles indicate the regions where the HAARP signal was detected for the two successful passes. All the trajectories were in the south-to-north direction.
Barr et al., 1985, 1986; Rietveld et al., 1989). Also, Getmantsev et al. (1974) performed the first ground-based observation of radiation at combination frequencies under HF heating, while Kotik and Trakhtengerts (1975) provided a theoretical treatment of this effect. In contrast to the many ground-based observations, space-based observations of HFgenerated ELF/VLF waves above the auroral electrojet are few in number. In this grouping we find observations concerning the heating facility of the Max-Planck Institut für Aeronomie (MPAe Heating) and the spacecraft AUREOL 3 (Lefeuvre et al., 1985), ISIS 1 (James et al., 1984) and DE 1 (Inan and Helliwell, 1985; James et al., 1990), all of them performed in December 1981. These experiments show that the modulated auroral electrojet can sometimes radiate detectable signals into the topside ionosphere and the magnetosphere. One of the important applications of the production of intense VLF waves which can propagate into the magnetosphere is the use of these signals for wave-injection experiments aimed at diagnostics studies of the magnetosphere (Helliwell, 1975; Kapustin et al., 1977; Stubbe et al., 1981, 1982).

The present paper reports recent observations on the Cluster spacecraft of electromagnetic ELF/VLF waves produced through ELF/VLF modulation of auroral electrojet currents. These observations were carried out over the ELF/VLF frequency range of $1068 \mathrm{~Hz}$ to $4375 \mathrm{~Hz}$ on the Cluster satellites SC3 and SC4, at a radial distance of $27000 \mathrm{~km}$ to $29000 \mathrm{~km}$. Figure 1 shows the geographical location of the Cluster passes during the times at which the experiment was performed, from August 2001 to June 2003. The successful receptions are highlighted in order to compare the ground track of the satellites with the location of the HAARP HF transmission facility near Gakona, Alaska. A numerical ray tracing model was used to simulate the propagation of VLF whistler mode waves from the ionosphere up to the spacecraft and to verify the propagation delay time at different frequencies.

\section{The Cluster experiment and the WBD instrument}

The Cluster spacecraft orbits traverse large regions of the magnetosphere. For the purpose of this paper, we focus on the perigee passes, when the four Cluster spacecraft travel closest to the Earth. The configuration of the four spacecraft near perigee can be either a tetrahedron or a "string of pearls" (Escoubet et al., 1997). The inter-spacecraft separation varied between 100 and $5000 \mathrm{~km}$ during the period of observations presented here. The HAARP-Cluster experiment was performed during passes around the magnetic equator at mid-altitude (between 4 and $6 \mathrm{Re}$ ). The observations of the HAARP transmissions were performed using the Cluster Wide Band (WBD) Plasma Wave Instrument (Gurnett et al., 1997). This instrument provides high-resolution frequency-time measurements of plasma waves in the Earth's magnetosphere. A digital wide-band receiver is used to provide electric or magnetic field waveforms over a wide range 
of frequencies. The WBD instrument transmits band-limited waveforms directly to the ground using a high-rate data-link. This direct transmission allows the recording of wideband waveforms for detailed high-resolution frequency-time analysis. Also, this technique has the advantage that the resolution can be targeted in post-processing to provide optimum analysis of the phenomena of interest.

\section{The High frequency Active Auroral Research Pro- gram (HAARP)}

The HAARP HF heater is located on a site near Gakona, Alaska. The geographic coordinates of the HF antenna array are approximately $62.4^{\circ}$ (north) latitude, $145.2^{\circ}$ (west) longitude. The geomagnetic coordinates for the facility are $63.1^{\circ}$ (north) latitude and $92.4^{\circ}$ (west) longitude. The principal instrument of the facility is a high power, HF phasedarray radio transmitter that is used to heat small, well-defined volumes of the ionosphere. The phased array is designed to transmit a narrow beam of high power radio signals in the 2.8 to $10 \mathrm{MHz}$ frequency range. At the time the experiments were performed, the HAARP HF antenna consisted of 48 elements arranged as a rectangular array of 8 columns by 6 rows. Each element making up the antenna array consists of four selectable dipole antennas, which are aligned north-south and east-west. The dipoles are mounted on an aluminum tower 72 feet high. There is one transmitter cabinet dedicated to each antenna mast. Each transmitter cabinet contains two identical transmitters, each of which is capable of producing a maximum output power of $10 \mathrm{~kW}$. A wire mesh ground screen is attached mechanically and electrically to the tower at a height of 15 feet above the ground (further information on the HAARP facility can be found at the website: www.haarp.alaska.edu). The HAARP transmitter had a total radiated power capability of $960 \mathrm{~kW}$ at the time the campaign described in this paper was performed. The ELF/VLF signal format shown in Fig. 2 was impressed upon the HF carrier through amplitude modulation. Two different HF carriers, $3.3 \mathrm{MHz}$ and $5.8 \mathrm{MHz}$, were used, with switching between carriers occurring every $10 \mathrm{~min}$. The HAARP HF transmitter requires $30 \mathrm{~s}$ to switch between carriers, thus the last half-minute of the 10-min cycle is used to change carriers, without transmitting during that period of time. The time required to change frequencies in the ELF/VLF band is approximately $20 \mu \mathrm{s}$.

\section{The HAARP-Cluster campaign}

The HAARP-Cluster campaign was conducted on six different occasions, 13 August 2001, 26 November 2001, 26 January 2003, 7 July 2003, 10 March 2003 and 11 May 2003, in two cases of which (26 January 2003 and 11 May 2003) detectable ELF/VLF signals were observed on one or more of the Cluster spacecraft. During these two dates the transmission schedule was as follows:

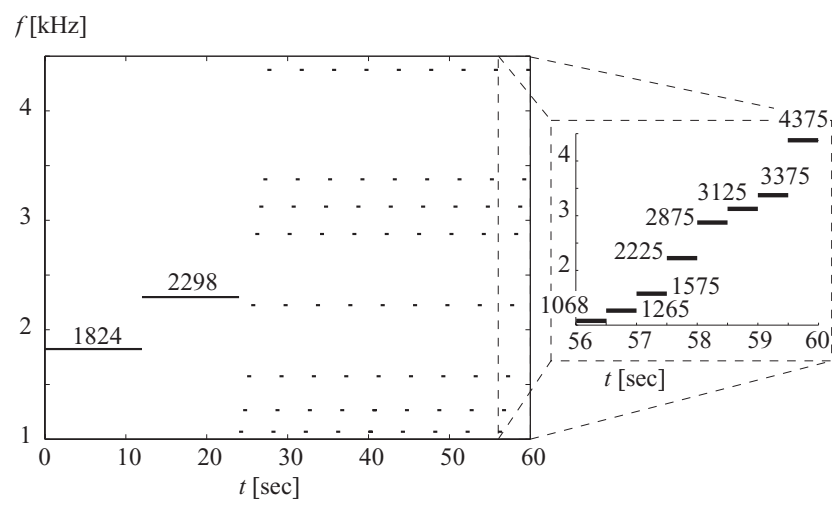

Fig. 2. Schematic spectrogram (to scale) of the transmission pattern used by the HAARP HF facility to heat the ionosphere. The ELF/VLF waves were AM modulated onto carrier frequencies of 3.3 MHz and $5.8 \mathrm{MHz}$, alternating between both. The term "Sinusoidal wave modulation" refers to the waveform used at each of the frequencies transmitted. The transmitted HF wave was in the $\mathrm{X}$ mode.

\section{January 2003: $12: 00-14: 00$ UT \\ 11 May 2003: 05:30-07:00 UT}

Figure 1 shows a detailed plot of the spacecraft orbital passes during these six dates, showing their azimuthal (Fig. 1a) and geographic (Fig. 1b) projection.

The HF carrier frequency was chosen to provide maximum heating in the D-region of the ionosphere (James et al., 1984), taking into account the allocated frequencies at which HAARP can transmit at its highest power. In this case the chosen frequency was $3.3 \mathrm{MHz}$, but transmissions also took place at $5.8 \mathrm{MHz}$ to test if there are differences in the emitted VLF radiation. According to Kapustin et al. (1977), and Stubbe et al. (1981, 1982), the maximum VLF radiated power can be achieved if the HF waves are polarized in the extraordinary $(\mathrm{X})$ mode; therefore, this particular polarization was chosen for HAARP transmissions. The HF beam was oriented vertically in order to have a transmitted HF wave with a $\boldsymbol{k}$-vector as perpendicular as possible to the $D$ layer of the ionosphere. The HF carrier was modulated sinusoidally in amplitude with a pattern of ELF/VLF waves, as shown in Fig. 2. The modulation pattern consists of two parts: first, two tones are transmitted for $12 \mathrm{~s}$ each, one at $1824 \mathrm{~Hz}$ and the other one at $2298 \mathrm{~Hz}$. These are followed by a staircase pattern of 8 frequencies, with each frequency being transmitted for $0.5 \mathrm{~s}$. This 4 -s pattern was repeated continuously throughout the remaining part of the minute. The one-min pattern was repeated throughout the duration of each Cluster pass. The HF carrier frequency was switched between $3.3 \mathrm{MHz}$ and $5.8 \mathrm{MHz}$ every $10 \mathrm{~min}$, in order to investigate the efficiency of ELF/VLF generation at different altitudes. After transmitting $9.5 \mathrm{~min}$ cycles at $3.3 \mathrm{MHz}, 30 \mathrm{~s}$ were required to change carrier frequency. Subsequent 9.5min cycles were then transmitted at 5.8 MHz. The ELF/VLF modulation frequencies were chosen in the range in which VLF generation was found in the past to be more efficient 

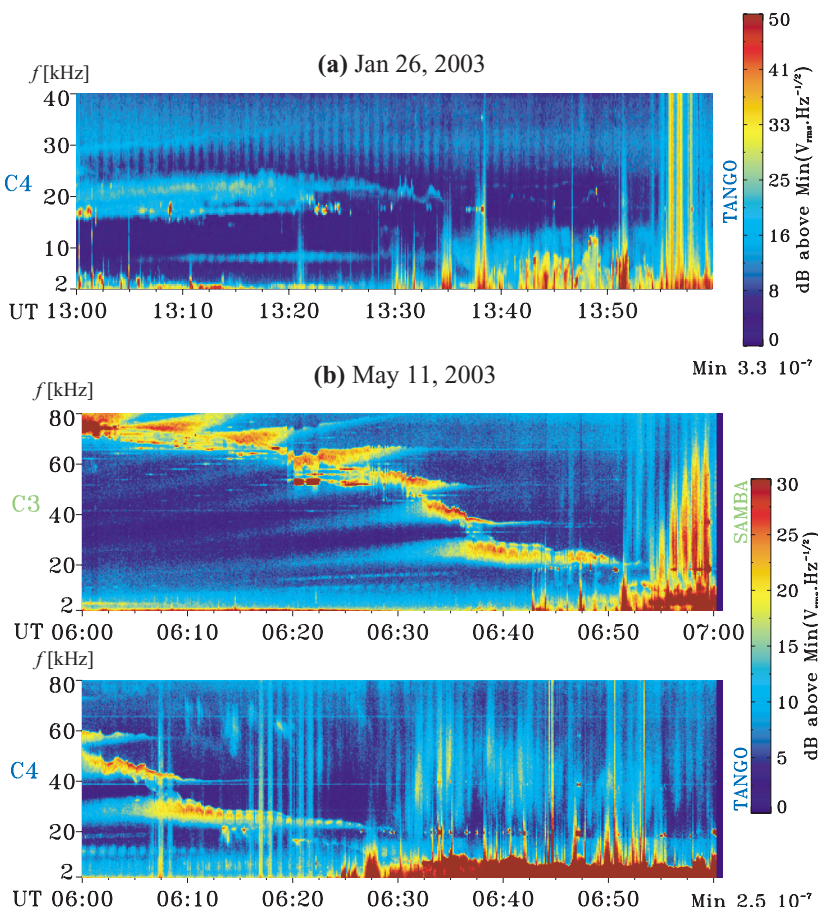

Fig. 3. WHISPER sounder electric field spectrograms for the two dates studied. The spectrograms show the intensity of the electric field. These three different days show a variety of electron density structures, from a very irregular plasma on the first panel to a very compressed plasmasphere on the second and third panels, where the plasma is smoother. Panel (a) corresponds to data recorded on 26 January 2003. Panel (b) corresponds to data recorded on 11 May 2003.

(Stubbe et al., 1981). The actual chosen values were not multiples of $60 \mathrm{~Hz}$, to avoid interference from harmonics of $60 \mathrm{~Hz}$ power grids (Helliwell et al., 1975). The duration of the pulses was chosen to be $0.5 \mathrm{~s}$, considered long enough to see temporal effects, such as rise time or possible triggered emissions (Helliwell et al., 1974), while still allowing for the sampling of different frequencies. The two initial long pulses of 12-s duration were designed to be detected by the Spatio Temporal Analysis of Field Fluctuations (STAFF) instrument on Cluster (Cornilleau-Wehrlin et al., 1997). This instrument uses a three-axis search coil magnetometer to measure magnetic fluctuations at frequencies up to $4 \mathrm{kHz}$, a waveform unit (up to either $10 \mathrm{~Hz}$ or $180 \mathrm{~Hz}$ ) and a Spectrum Analyzer (up to $4 \mathrm{kHz}$ ). The time resolution for a complete multicomponent waveform measurement varies between $0.125 \mathrm{~s}$ and $4 \mathrm{~s}$.

\section{Simulation results: the ray-tracing technique}

We performed ray-tracing calculations to determine the propagation paths and to estimate the time delays of the HAARP VLF signals received on Cluster. The ray-tracing method used is that which was introduced by Haselgrove (1954) and Yabroff (1961), with the original software implemen-
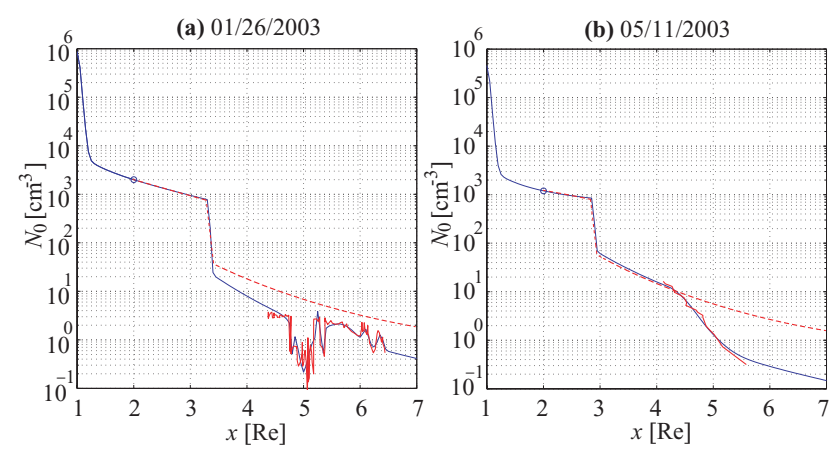

Fig. 4. Electron density profiles derived from the WHISPER electric field measurements for the two dates studied. These are constructed from direct measurement of the plasma frequency using WHISPER data at different magnetic latitudes and then projected to the equator, assuming an $R^{-4}$ variation of the electron density with radial distance from the Earth. (a) Profile for 26 January 2003. (b) Profile for 11 May 2003. The red full line is the electron density measured, while the red dashed line is the model. The blue line represents the actual electron density profile used in the simulations.

tation undertaken by Kimura (1966). The actual version of the Stanford ray-tracing program used for the computations presented here is described elsewhere (Inan and Bell, 1977). We used a geomagnetic field model based on a centered dipole with electron gyrofrequency of $880 \mathrm{kHz}$ at the ground on the magnetic equator. The electron and ion densities above $1000 \mathrm{~km}$ are represented by a field-aligned isothermal $\left(\mathrm{T}=1600^{\circ} \mathrm{K}\right)$ diffusive equilibrium model for regions within the plasmasphere $(L \leq 4)$. At higher $L$-shells the electron density profile was assumed to vary as $R^{-4}$, where $R$ is the radial distance from the Earth. At $1000 \mathrm{~km}$ the ionospheric composition was taken to be $90 \% \mathrm{O}^{+}, 8 \% \mathrm{H}^{+}$and $2 \% \mathrm{He}^{+}$. The same composition was used in both cases where the HAARP signal was detected in Cluster.

Data from the WHISPER resonance sounder instrument (Décréau et al., 1997) was used to deduce the electron density profile. The WHISPER sounder on the Cluster spacecraft is designed to provide an absolute measurement of the total plasma density $N_{o}$ within the range $0.2-80 / \mathrm{cm}^{3}$, which is the range of interest for the HAARP-Cluster experiment. WHISPER performs the identification of the electron plasma frequency by analyzing the pattern of resonances triggered in the medium by a pulse transmitter. This instrument uses a resonance sounding technique, already proved successful in the regions to be explored by previous experiments (Etcheto et al., 1983; Trotignon et al., 1986). A Fast Fourier Transform (FFT) calculation provides the wave analysis function of the instrument. In the basic nominal operational mode, which is the one we present in this paper, the density is measured every $28 \mathrm{~s}$, the frequency and time resolution for the wave measurements are about $300 \mathrm{~Hz}$ and $2.2 \mathrm{~s}$. Figure 3 shows representative frequency-time spectrograms generated from measured data using WHISPER for the two cases studied. The two panels show spectrograms of the electric field along the $z$ axis (i.e. in the spin plane of 
the spacecraft). Figure 3 a displays the $\mathbf{E}_{z}$ field on spacecraft SC3, on 26 January 2003. Figure $3 \mathrm{~b}$ consists of two spectrograms, both measurements of the $\mathbf{E}_{z}$ field, the first one on spacecraft SC3, and the second one on spacecraft SC4, for the pass on 11 May 2003. Data shown in these three spectrograms were recorded using the WHISPER instrument in the passive mode (Décréau et al., 1997). The plasma resonances (not shown here) that can be excited when WHISPER is active are those at the electron plasma frequency, the electron gyro-frequency and its harmonics, and the upper hybrid frequency. A number of Bernstein waves are also often excited (Bernstein, 1958). In the passive mode, natural wave bands between two successive harmonics of the electron gyro-frequency can be observed. We used these active and passive measurements to obtain the electron density through the method specified by Canu et al. (2001) and Trotignon et al. $(2001,2003)$. The density derived from the local measurement provided by WHISPER along the spacecraft orbit was used to derive the density along $L$ shells using an $R^{-4}$ model for the electron density (Persoon et al., 1983; Gallagher et al., 2000). Since the spacecraft cross the magnetosphere at different $L$ shells and magnetic longitudes, the derived electron density is a function of the satellite position. We can then project this electron density to the magnetic equator, assuming that the electron density profile varies as $R^{-4}$, where $R$ is the radial distance from the Earth. The projection is displayed in Fig. 4, which shows the resulting density profiles at the equator for the two days. The results are shown superimposed upon the electron density profile obtained from the Carpenter and Anderson (1992) model. The irregularities in the electron density profile are assumed to be field-aligned enhancements of ionization and are included in the ray-tracing code by multiplying the densities given by the $R^{-4}$ model, with a product of bell-shaped functions for each enhancement:

$N_{0}=N_{D E} \cdot \prod_{i=1}^{m}\left\{1+C_{i} \cdot \exp \left(\frac{-\left(L-L_{i}\right)^{2}}{2 \cdot \Delta L_{i}^{2}}\right)\right\}$,

where

$N_{0} \quad=$ Plasma electron density at the equator

$N_{\mathrm{DE}}=$ Plasma electron density derived from model

$m \quad=$ number of enhancements

$C_{i} \quad=$ enhancement at i-th duct

$L \quad=$ Local $L$-Shell

$L_{i} \quad=L$-Shell at center of $\mathrm{i}$-th duct

$\Delta L_{i} \quad=$ semi-width of $\mathrm{i}$-th duct.

Following the measured densities obtained from WHISPER, these enhancements or troughs were added for each case, with the coefficients $C_{i}, L_{i}$, and $\Delta L_{i}$ chosen to fit the data.

The density profiles obtained as described above are used as inputs for the ray-tracing code. The smooth, solid line represents the calculations based on the Carpenter and Anderson (1992) model. Superimposed on top are the values of electron density obtained from the WHISPER instrument as projected to the equator. Figures $4 \mathrm{a}$ and $\mathrm{b}$, respectively,
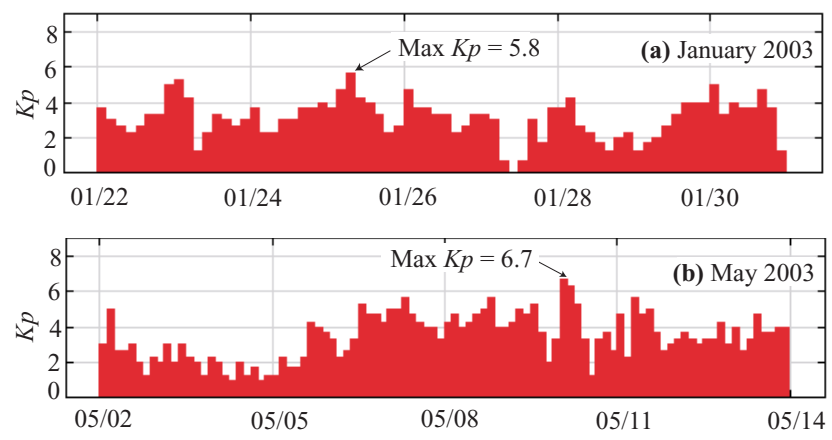

Fig. 5. Average planetary $K_{p}$ index, as recorded for dates studied. (a) Display of the $K_{p}$ index during the end of January. (b) Display of the $K_{p}$ index during the beginning of May. Each value of $K_{p}$ represents a 3 -h average number derived from the standardized $K$ index $(K s)$ of 13 magnetic observatories.

show the resulting density profiles for 26 January 2003 and 11 May 2003. On this plot, we can see the equatorial density profile over the $L$ shell range for which the electron density $N_{o} \leq 80 \mathrm{el} / \mathrm{cm}^{3}$. For regions of higher electron density, we use the model of Carpenter and Anderson (1992) to complete the profile at lower altitudes (i.e. $R<4$ ), where no WHISPER data is available. It should be noted that the electron density model proposed by Carpenter and Anderson (1992) does not reflect the actual density profile in detail, but rather specifies an average profile, since the actual profile depends on the maximum $K_{p}$ index of the previous $24 \mathrm{~h}$. The maximum $K_{p}$ indices for the three days used in the Carpenter and Anderson model, are: 26 January $2003-\max K_{p} \sim 6,11$ May $2003-$ $\max K_{p} \sim 7$. Figure 5 shows the variation of the geomagnetic activity during the relevant time periods, including the dates before and after the events of the experiments. This characteristic is important in interpreting the results. We notice that the $K_{p}$ index was very high during both successful passes, reaching a local maximum at that time.

The results of the ray tracings are displayed in Fig. 6. The red lines are the paths of the rays at $3.125 \mathrm{kHz}$ for different initial wave normal angles $\psi$ at the point of injection into the magnetosphere, at an altitude of $1000 \mathrm{~km}$ above the surface of the Earth. Highlighted are the satellite trajectories and the region of detection of the HAARP signal. Figure 6a correspond to the 26 January 2003 case, when the signal was detected only for $5 \mathrm{~s}$, for which the region of illumination is highlighted with a star $(*)$. Figure $6 \mathrm{~b}$ corresponds to the 11 May 2003 case, when the signal was detected for $15 \mathrm{~min}$, and the region of illumination is highlighted with a red dotted line. The background color quantitatively represents the electron density, derived using an $R^{-4}$ model (Persoon et al., 1983) to project the measured density obtained from the WHISPER instrument along the satellite trajectory to other latitudes different from the satellite path. According to these simulations, the rays are injected into the magnetosphere very close to the latitude of the HAARP facility, between $64^{\circ}$ (on 11 May 2003) and $63^{\circ}$ latitude (on 26 January 2003), at 
(a) $01 / 26 / 2003$. Rays launched from $\lambda_{\mathrm{m}}=63^{\circ}$, @ $-5^{\circ}<\psi<5^{\circ}$

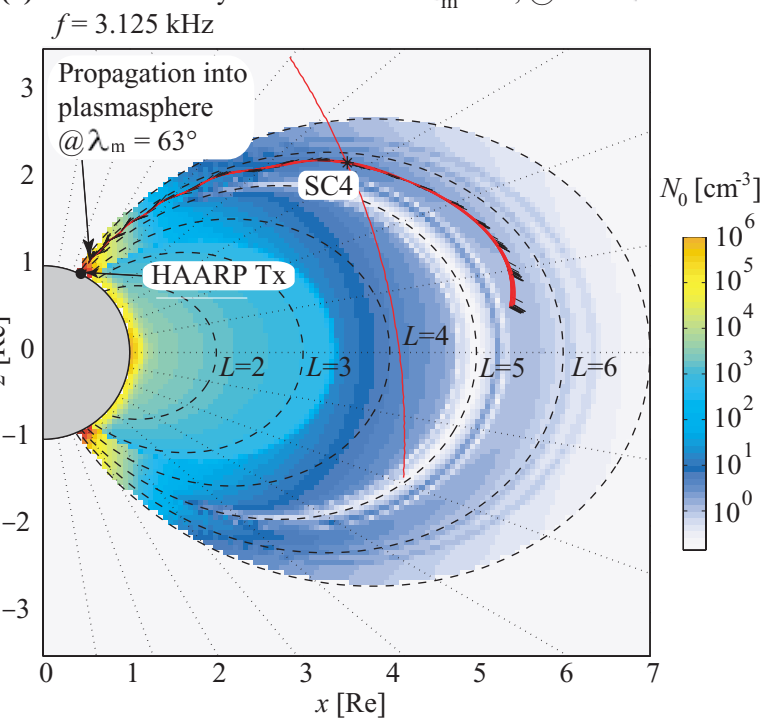

(b) $05 / 11 / 2003$. Rays launched from $\lambda_{\mathrm{m}}=64^{\circ}$, @ $5^{\circ}<\psi<7^{\circ}$

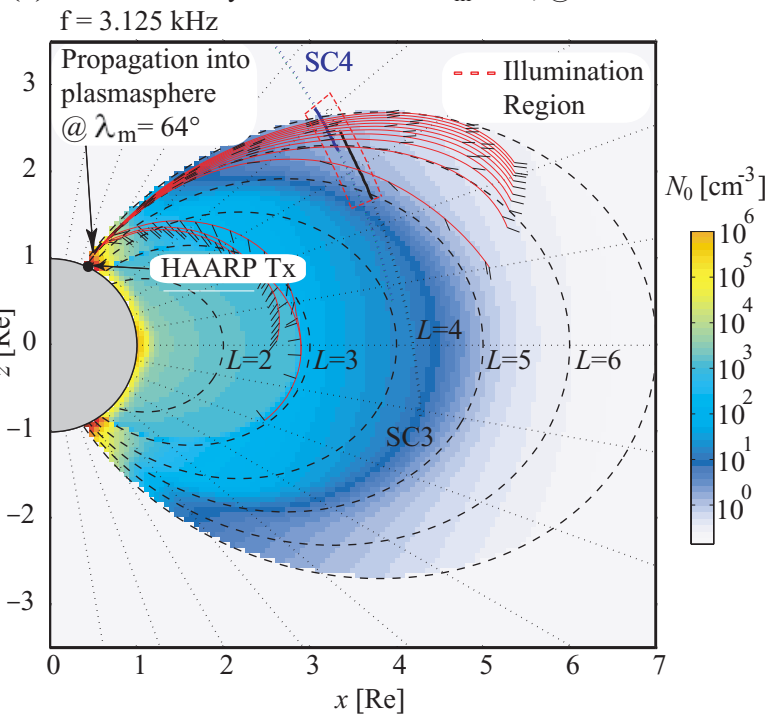

Fig. 6. Ray-tracing simulation of the wave propagation path for the two dates studied. Panel (a) shows ray paths from the simulation corresponding to 26 January 2003. Panel (b) shows ray paths from the simulation corresponding to 11 May 2003. Rays were launched at $3.125 \mathrm{kHz}$ from different latitudes on the ground, at locations near the HAARP facility and for different wave normal angles at the launching point, where the wave is injected into the magnetosphere. The color scale represents the value of plasma density as measured from WHISPER and projected into the 2-D meridional plane using a diffusive equilibrium model in the plasmasphere and an $R^{-4}$ in the magnetosphere outside the plasmasphere. Also plotted is the trajectory of the spacecraft and the region where the HAARP signal was detected. The ray paths are displayed as a thin red line, and the direction of the $\boldsymbol{k}$ vector is shown as a short black line periodically spaced along the path. Notice the location of the HAARP facility with respect to the point of injection of the wave into the magnetosphere.

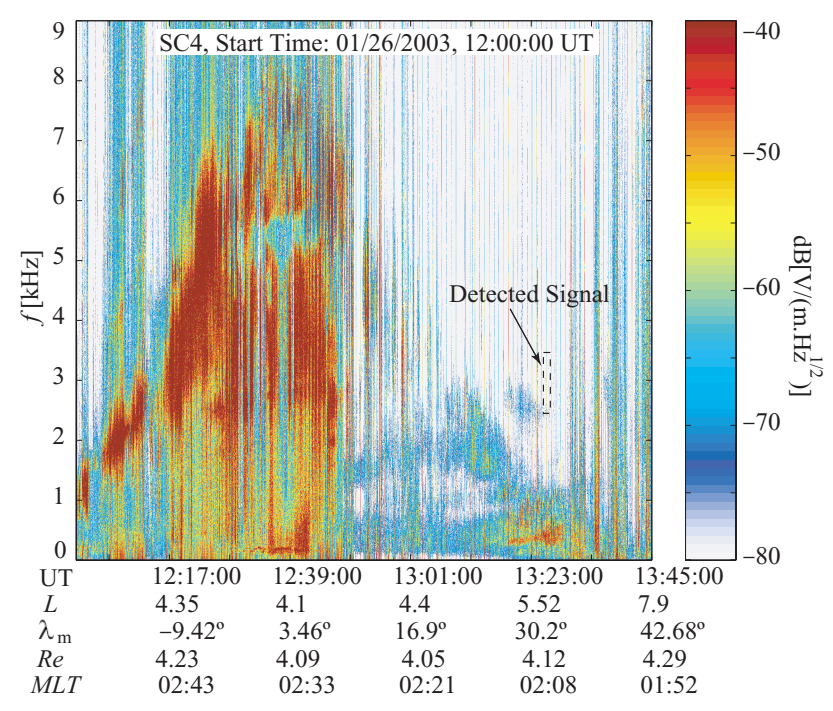

Fig. 7. WBD overview spectrogram from the Cluster satellite pass during 26 January 2003, starting at 12:00:00 UT. The magnitude scale refers to electric field intensity,. The time and frequency range in which the HAARP signal was detected during this date is indicated by a dashed-line rectangle. Information about the location of the spacecraft, the $L$ shell, Magnetic Local Time (MLT) the radial distance to the Earth $(\mathrm{Re})$ and magnetic latitude $\left(\lambda_{m}\right)$ is shown below.

a distance of $\sim 200 \mathrm{~km}$ east of HAARP. Figure 6a shows the simulation for 26 January 2003 where we sampled the initial wavenormal angle in a range from $-20^{\circ}<\psi<20^{\circ}$. We found that even a small change in the initial wave-normal angle can cause the rays to diverge in latitude and not reach the satellite. A refined calculation using smaller values of $\psi(-$ $5^{\circ}<\psi<5^{\circ}$ ) at the same latitude of injection shows that the signals can reach the spacecraft only if the rays are confined within a cone of $\pm 5^{\circ}$ about the input wave normal angle of $0^{\circ}$.

Following this method for the 11 May 2003 case we traced different rays at different initial values of $\psi$ to determine the location of injection of the waves into the magnetosphere. We find that the waves were most likely injected at latitude of $64^{\circ}$, as displayed in Fig. 6b. If we refine the calculation using smaller increments in $\psi\left(5^{\circ}<\psi<7^{\circ}\right)$, at the same latitude of injection, we find that the signals can reach the spacecraft only if the rays are confined within a cone of $\pm 1^{\circ}$ about the input wave normal angle of $6^{\circ}$. In addition, it was found that the injection latitude of the rays that reached Cluster was confined to a $1^{\circ}$ range. A comparison with this figure shows that the maximum deviation from this value can be $0.5^{\circ}$. Therefore, we find that for $\mathrm{f}=3.125 \mathrm{kHz}$, the location of the injection point of the rays which reach the spacecraft is at latitude of $63^{\circ} \pm 1^{\circ}$ for 26 January 2003 and $64^{\circ} \pm 0.5^{\circ}$ for 11 May 2003. The rays enter the magnetosphere $\sim 200 \mathrm{~km}$ west of the HAARP location, and we assume that the ELF/VLF wave energy has propagated in the Earth-ionosphere wave guide in order to reach the entry point. Thus, the intensity of the input 


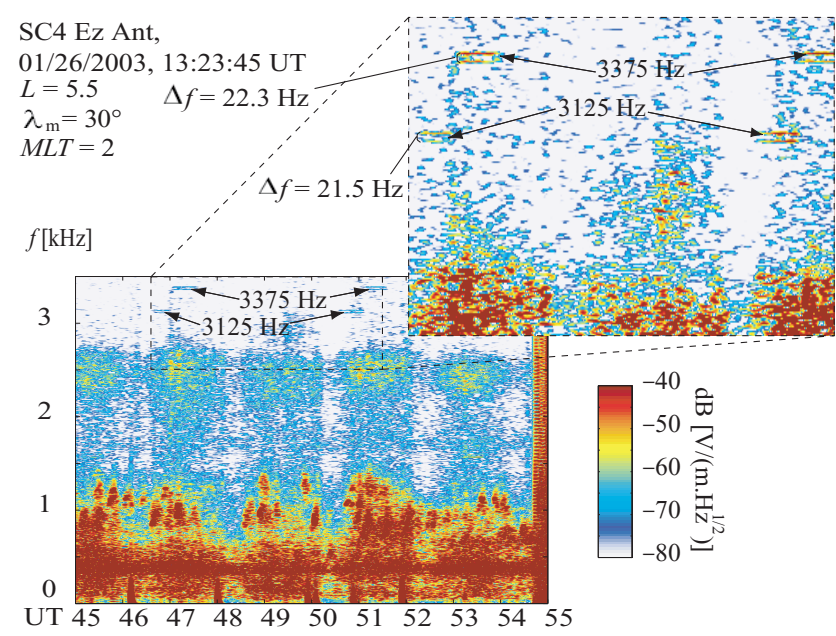

Fig. 8. Detailed WBD spectrogram from the Cluster satellite pass during 26 January 2003, at the time of detection (13:23:45 UT), of the HAARP signal. The magnitude scale refers to electric field intensity. Highlighted are the detected pulses and the separation between the two sideband waves in the detailed zoom-in. Information about the location of the spacecraft, the $L$ shell, Magnetic Local Time (MLT) the radial distance to the Earth (Re) and magnetic latitude $\left(\lambda_{m}\right)$ is shown below.

waves does not directly give definitive information about the intensity of the actual source of the ELV/VLF waves, since propagation loss in the Earth-ionosphere wave guide must be taken into account, as well as the efficiency of coupling of the source radiation into the wave guide. The ray tracings for both dates show that the extent of the illuminated region (in the direction perpendicular to the magnetic field line in the meridional plane) in the magnetosphere can vary from $\sim 6500 \mathrm{~km}$, as we can see in Fig. $6 \mathrm{~b}$, to $\sim 500 \mathrm{~km}$ as we can notice in Fig. 6a. This difference in size of the corresponding illuminated regions for both dates is directly related to the possibility of the ray paths being "ducted" along the field lines when the plasma density is very irregular (as in 26 January 2003, see Fig. 4a) and when the plasma density is not so irregular (as in 11 May 2003, see Fig. 4b). Ray-tracing calculations suggested that the propagation time of the signals from the source to the spacecraft was $\sim 0.18 \mathrm{~s}$ for 26 January 2003 and $\sim 0.22$ s for 11 May 2003.

\section{Observations: the HAARP signal detected at Cluster with the WBD instrument}

As mentioned previously, the HAARP transmission was detected on 26 January 2003 between 13:24:46 UT and 13:24:51 UT, on spacecraft SC4 and on 11 May 2003 between 06:30:00 UT and 06:52:00 UT on spacecraft SC3 and between 06:12:00 UT and 06:27:00 UT on spacecraft SC4. Thus, ELF/VLF waves generated by modulated electrojet currents were observed on one-third of the satellite passes

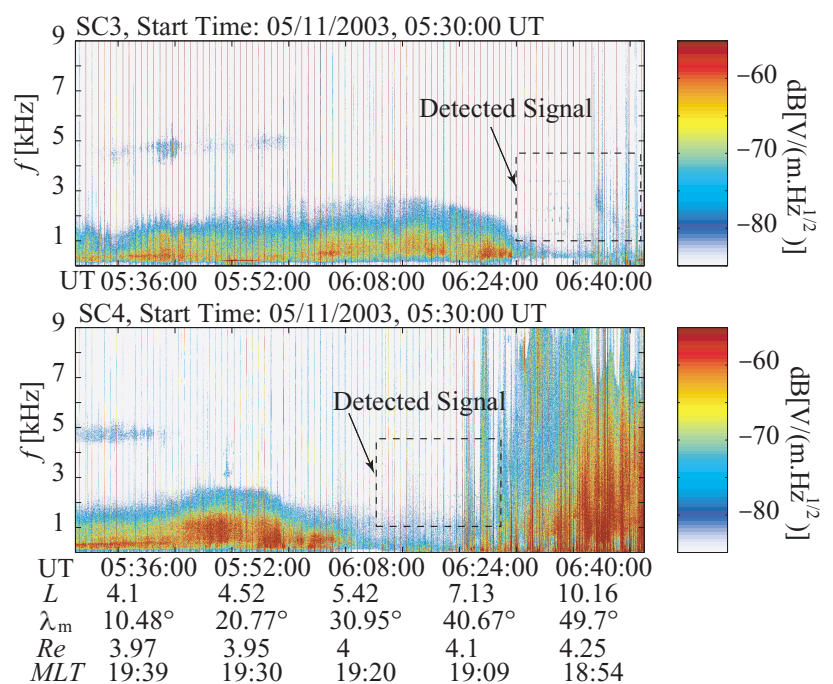

Fig. 9. WBD overview spectrogram from the Cluster satellite pass during 11 May 2003, starting at 05:30:00 UT. The magnitude scale refers to electric field intensity. The time and frequency range in which the HAARP signal was detected during this date is indicated by a dashed-line rectangle. Information about the location of the spacecraft, the $L$ shell, Magnetic Local Time (MLT) the radial distance to the Earth $(\mathrm{Re})$ and magnetic latitude $\left(\lambda_{m}\right)$ is shown below.

scheduled to observe them. There are several possible explanations for this variability, the two most important ones being the intensity of the electrojet and the ambient ELF/VLF wave activity level in the magnetosphere. Indeed, as noted in previous similar experiments (Lefeuvre et al., 1985; James et al., 1984; Inan and Helliwell, 1985), the intensity of the $\mathrm{ELF} / \mathrm{VLF}$ radiation is expected to be higher when the intensity of the electrojet is higher. This condition imposes a degree of uncertainty for the experiment, because it then depends on external conditions that cannot be controlled. The same argument can be applied to the second explanation. If there are intense ELF/VLF natural plasma waves in the region of space within which the spacecraft moves at the time of transmissions, the HAARP generated waves might be "obscured" by these natural waves (by causing the receiver automatic gain setting to be lower), and not readily discernible in the data.

Figure 7 shows an overview spectrogram of data recorded using the WBD instrument during 26 January 2003. Highlighted is the time of detection of the HAARP signal. Figure 8 shows a detailed spectrogram for the period of detection of the signal on this date. Two of the sequences of ELF/VLF pulses generated by the HAARP heater are visible in this record. The two pulses detected here correspond to the ones generated at $3.125 \mathrm{kHz}$ and $3.375 \mathrm{kHz}$. Closer examination of the detected pulses shows that at both frequencies there actually are two pulses roughly centered close to the nominal frequency, separated in frequency by approximately $25 \mathrm{~Hz}$. These sideband waves have been interpreted as being lower hybrid (LH) waves possibly generated through linear 
mode coupling in the presence of small-scale plasma density irregularities (Bell and Ngo, 1990; Bell et al., 2004).

Results from 11 May 2003 reveal more extensive sideband wave structure. On this day, the signal was detected for at least $15 \mathrm{~min}$, and the natural ELF/VLF emissions that are usually present in these regions of the magnetosphere were not present, therefore providing a much better environment for identification of the HAARP ELF/VLF signals. Figure 9 shows data recorded using the WBD instrument on 11 May 2003. Highlighted is the time of detection of the HAARP signal. Figure 10 shows a detailed spectrogram of the period of detection of the signal on this date. The full sequences of ELF/VLF pulses generated by the HAARP heater over the whole range of frequencies are clearly detected on Cluster. Figure 10a shows the characteristics of the HAARP signals detected during this period, including the long 12-s pulse at $1824 \mathrm{~Hz}$, and the 2nd harmonics of some of the signals that are generated by the HAARP HF heater. Figure $10 \mathrm{~b}$ displays a detailed spectrogram of the 12 -s pulse at $1824 \mathrm{~Hz}$. This spectrogram reveals a periodic amplitude variation of 2-s period, which is due to "spin fading". As the antenna rotates, its orientation with respect to the wave E-field changes, since the signal in the receiver is proportional to the projection of the wave E-field along the antenna. This effect produces a periodic fade, called spin fading, affecting all of the measurements that are performed using the electric antenna.

A very interesting feature that is also displayed in Fig. 10a, $\mathrm{b}$ and $\mathrm{c}$ are the additional pulses of similar duration as the original pulse, centered close to the nominal frequency, separated by approximately $25 \mathrm{~Hz}$. These are similar to the sideband waves shown in Fig. 8. These sideband waves can be explained as the result of the antenna response to short wavelength lower hybrid waves, as discussed in detail by Bell et al. (2004). The value of the spacing between the sideband waves can be seen from Fig. 11, where the power spectrum is plotted every $0.1 \mathrm{~s}$ after 06:37:53 UT. It is very clear how the spacing between sidebands is always $\sim 25 \mathrm{~Hz}$.

According to Helliwell et al. (1975), waves with frequencies corresponding to the harmonics of the $60 \mathrm{~Hz}$ power line frequency can be detected in space and subharmonics of $60 \mathrm{~Hz}$ are also observed. However these waves generally endure for times much longer than the $0.5 \mathrm{~s}$ duration of the HAARP generated ELF/VLF pulses. Thus we believe it to be highly unlikely that the side band waves are related to harmonic radiation from the power grid.

In order to verify that the sideband waves were not somehow generated from the modulation of the electrojet by the HAARP signal, we carried out ELF/VLF observations on the ground near Chistochina in Alaska, at a distance of $\sim 36 \mathrm{~km}$ from the HAARP HF heater. The ELF/VLF receiver at this site recorded the signal emitted from the ionosphere in the band of interest. The results of these measurements are shown in the spectrograms of Fig. 12. The data displayed in this figure are measurements of the magnetic field as received by two loop antennas oriented perpendicular with respect to each other, respectively in the geographic north-south and east-west directions. In both measurements the scale

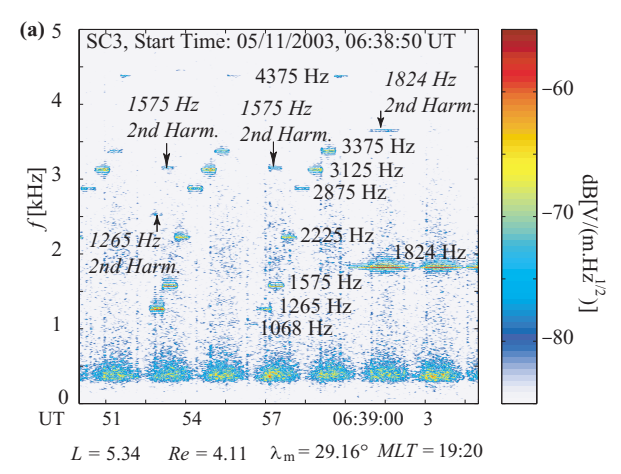

(b) SC3, Start Time: 05/11/2003,06:38:00 UT
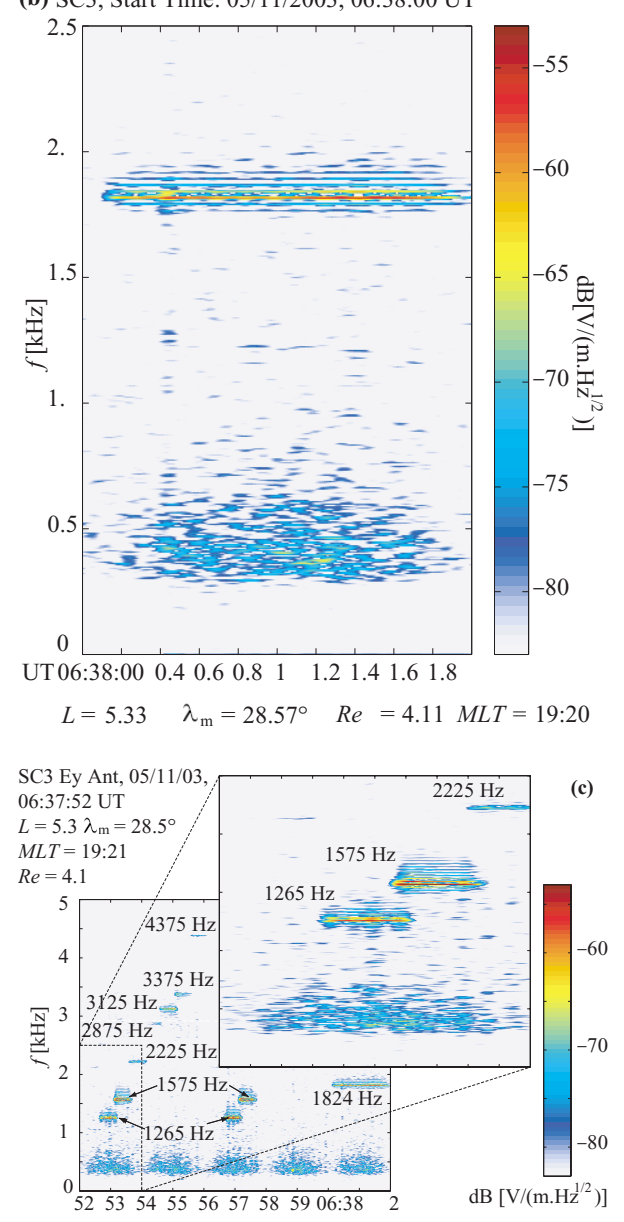

Fig. 10. Detailed WBD spectrograms from the Cluster satellite pass during 11 May 2003. (a) An overview of the detected HAARP signal, including the harmonics generated by the HF heater. The whole transmitted pattern at all frequencies is observed, as well as sideband waves appearing around the stronger pulses. (b) An overview of the detected HAARP signal at $1824 \mathrm{~Hz}$, showing sideband waves generated around the long 12-s pulse and the characteristic "spin fade". (c) Detailed spectrogram of two of the stronger pulses at frequencies $1265 \mathrm{~Hz}$ and $1575 \mathrm{~Hz}$. The zoom-in shows the sideband waves, which extend several hundred $\mathrm{Hz}$ above and below the nominal frequency. Also notice the spin modulated baseband noise. The magnitude scale refers to electric field intensity. 
(a) 6:37:53 UT
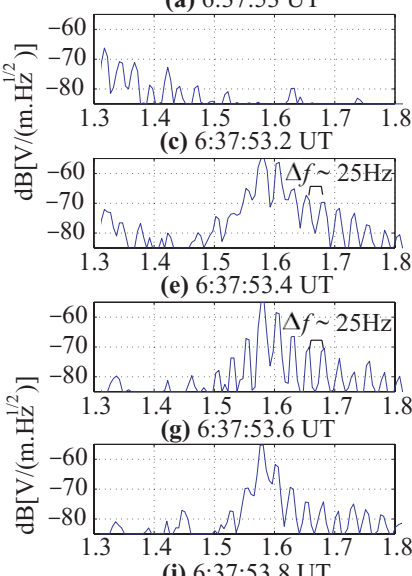

(i) $6: 37: 53.8 \mathrm{UT}$

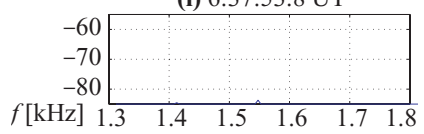

(b) $6: 37: 53.1 \mathrm{UT}$
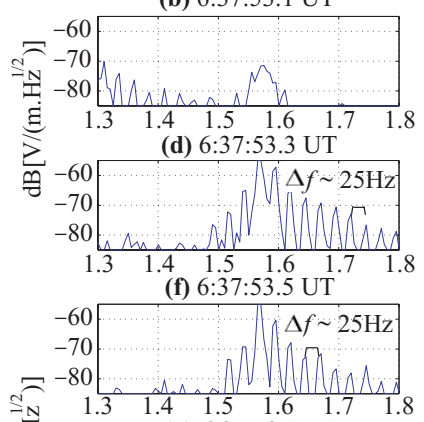

(h) $6: 37: 53.7 \mathrm{UT}$

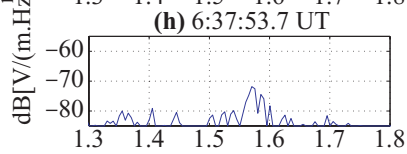

(j) $6: 37: 53.9$ UT

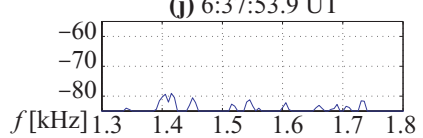

Fig. 11. Detailed power spectrum of the 0.5-s pulse detected on spacecraft SC3, at 06:37:53 UT. Each of the ten panels, from (a) to (j) correspond to a different spectrum obtained at time intervals of $0.1 \mathrm{~s}$. Highlighted in panels (c), (d), (e) and (f) is the separation between sidebands, of $\sim 25 \mathrm{~Hz}$. The magnitude scale refers to electric field intensity.

reference is absolute in $\mathrm{dB}[\mathrm{pT}]$. This type of data provides an indication of the strength of the ELF/VLF signal generated by the auroral electrojet antenna. However, the directional properties of the radiation are not well known, so that the radiation directed upward into the magnetosphere may be significantly different from that directed into the Earthionosphere waveguide. Figure 12a displays the signal detected in Chistochina during the 26 January 2003, pass. In this case the magnetometer at the HAARP site showed evidence of a very intense electrojet current overhead. At the same time, very intense HAARP generated ELF/VLF waves were detected at Chistochina. The same situation can be noted in Fig. 12b, where the signals received on the ground during the 11 May 2003 pass are as intense as the ones received on 26 January 2003. In this case also, HAARP magnetometer data showed evidence of an intense electrojet current overhead.

Finally, we should also mention that while the 12 pulses at $1824 \mathrm{~Hz}$ and $2298 \mathrm{~Hz}$ were transmitted in order to be detected by the STAFF instruments on Cluster, the HAARP signal was not detected by this instrument, possibly due to either the long integration times required or to the fact that LH waves possess only very small magnetic fields.

\section{Conclusions}

The Cluster-HAARP experiments are opening new possibilities in the field of magnetospheric probing using ELF/VLF wave-injection. Ray-tracing simulations suggest that the scale of the illuminated region in the magnetosphere can
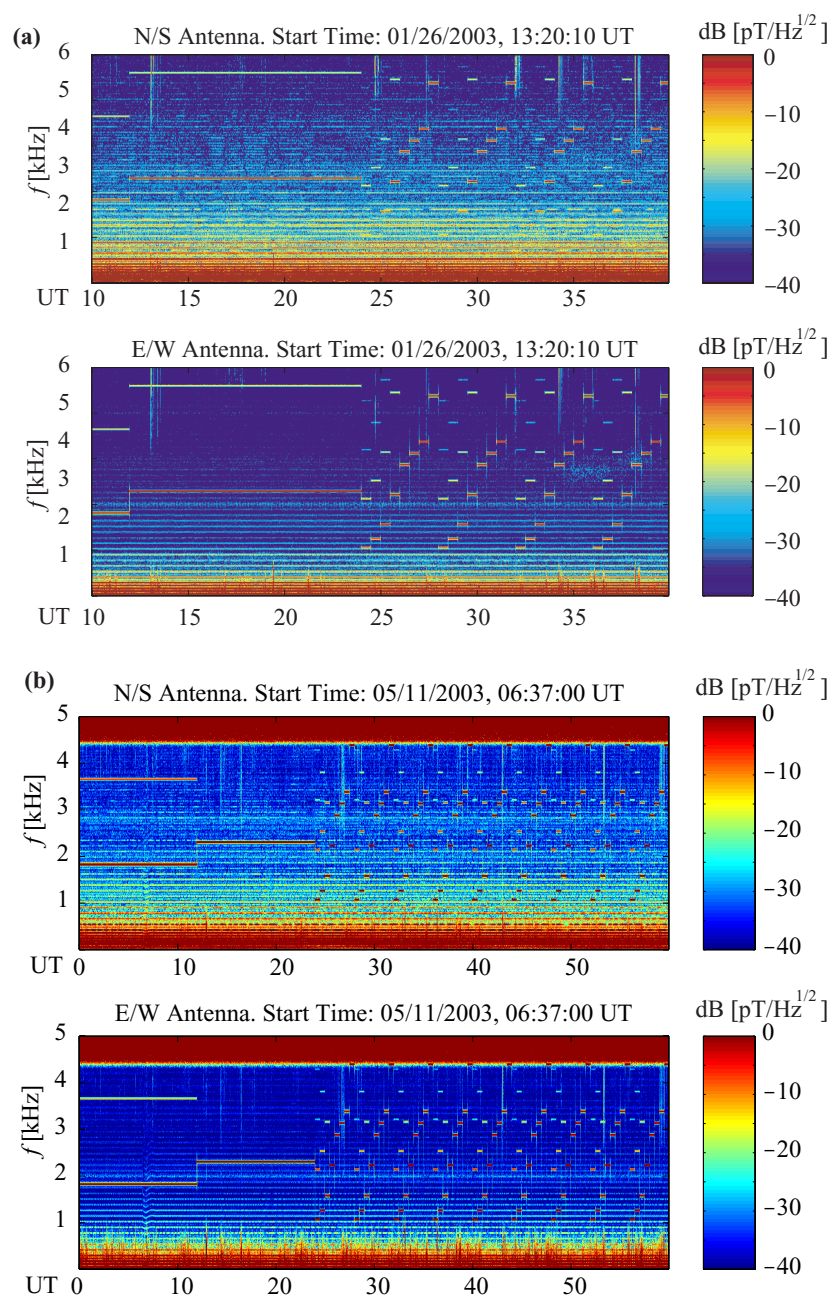

Fig. 12. Ground observations of the ELF/VLF HAARP generated signal at Chistochina. (a) Measured signal during 26 January 2003, starting at 13:20:10 UT. (b) Measured signal during 11 May 2003, starting at 06:37:00 UT. Each panel in both displays corresponds to a different magnetic loop antenna, one being oriented to the geographic north-south line and the other one oriented to the geographic east-west line. In both cases strong ELF/VLF signals were recorded on the ground, indicating the presence of modulated electrojet currents in the ionosphere. The magnitude scale refers to magnetic field intensity in $\mathrm{pT}$.

vary from $\sim 6500 \mathrm{~km}$, (for the experiment on 11 May 2003) to $\sim 500 \mathrm{~km}$ (for the experiment on 26 January 2003). We clearly cannot make definitive conclusions about the extent of the source region in the ionosphere, since the ground track of the Cluster spacecraft orbits do not cross directly over the HAARP illuminated region, but rather west of it $(\sim 200 \mathrm{~km})$. The data obtained from the HAARP-Cluster campaign provides experimental evidence of the excitation of quasi-electrostatic lower hybrid waves (Bell et al., 2004) by the HAARP generated electromagnetic whistler mode waves as evidenced by the sideband waves detected with most of the pulses on 11 May 2003. As the whistler mode waves propagate through the highly irregular region 
immediately outside the plasmapause, they apparently continuously lose energy to the lower hybrid waves. This energy loss may explain the lack of lightning-generated whistlers in the region outside the plasmasphere, as pointed out by Platino et al. (2002).

Ray-tracing simulations were used to determine some of the characteristics of the HAARP generated ELF/VLF waves, such as the latitude of the injection of the ELF/VLF wave into the magnetosphere, and the estimated path and time of travel from the Earth to the spacecraft. The results suggest that the rays were injected between $63^{\circ} \pm 1^{\circ}$ and $64^{\circ} \pm 0.5^{\circ}$ in latitude with wave-normal angles ranging from $-5^{\circ}$ to $5^{\circ}$ on the two days the HAARP-generated ELF/VLF signals were detected. The propagation time of the wave from the source to the spacecraft was predicted to be of about $0.18 \pm 0.01 \mathrm{~s}$ and $0.22 \pm 0.01 \mathrm{~s}$ for the two days. For the 26 January 2003 case, we compared the times of arrival measured on the received ELF/VLF signal at Cluster, with the time of transmission at HAARP. Subtracting these two values we obtain a measured value of $0.18 \pm 0.01 \mathrm{~s}$ for the propagation time from the ionosphere to the satellite. In this connection, it should be noted that the propagation time of the heater signal from the ground to the ionosphere is only a few milliseconds and is thus negligible compared to the propagation time from the ionosphere to the spacecraft. Comparing the measurements on the ground with those measured on the spacecraft in the 11 May 2003 case we find a measured propagation time of $0.22 \pm 0.01 \mathrm{~s}$, in reasonable agreement with the ray-tracing results. This measurement takes into account the propagation time of the signal from the heated region to the ground station, which is estimated to be $3 \mathrm{~ms}$. The uncertainty of the measured delay also has to take into account the finite $\Delta t$ value used in preparation of the spectrogram, which was $0.01 \mathrm{~s}$ for the ground-based data and $0.005 \mathrm{~s}$ for the Cluster data.

There are some important factors to be considered for any future experiments: The $K_{p}$ magnetic indices were relatively high on the two dates of successful detection of the HAARP signals at the Cluster spacecraft. This fact suggests that high magnetic activity might be a necessary condition for successful detection of HAARP-generated ELF/VLF waves beyond $L \sim 4$. This conclusion can be tested with statistical analysis, which requires the completion of more successful passes. High magnetic activity is generally associated with stronger electrojet currents, and when these larger currents are modulated by HAARP, they may radiate more intense ELF/VLF signals into the magnetosphere.

The other important factor noticed is the fact that the electron densities on these two days were up to an order of magnitude lower than the ones found for lower $K_{p}$ indices of $\sim 3$ for the $L$-shells range covered by the Cluster passes shown in Fig. 1a, i.e. $4<L<6$. Evidence of ducting was not found, and the densities were significantly different on both days in terms of irregularities.

The HAARP HF heater used in these experiments is now in the process of being expanded into an array four times as large as the present array. The total radiated HF power will be increased approximately by a factor of four. It is expected that the expanded array will be able to more strongly modulate electrojet currents and to produce more intense ELF/VLF waves. Thus, the possibility for magnetospheric probing by HAARP-generated ELF/VLF waves can be expected to be greatly enhanced.

Acknowledgements. This work was supported, in part, by the High Frequency Active Auroral Research Program (HAARP), under Grant N00014-03-0631 from the Office of Naval Research (ONR). This research was also supported in part by the National Aeronautics and Space Administration (NASA), under parent grant NAG5-9974 at the University of Iowa and with Subcontract No. 4000061641 to Stanford University. We greatly appreciate the help of C. Abramo of DSN and M. Hapgood of RAL in scheduling the real-time wideband data acquisition from the WBD receiver. We thank D. Gurnett (PI for WBD) for his support and P. Décréau who provided the WHISPER data. We thank R. Moore and M. Golkowski for providing the Chistochina data. We also thank P. A. Kossey for his support in the use of the HAARP facilities and M. J. McCarrick for setting up the transmissions schedule and timefrequency patterns in the HAARP HF heater.

Topical Editor T. Pulkkinen thanks G. James and another referee for their help in evaluating this paper.

\section{References}

Barr, R. M., Rietveld, M. T., Kopka, H., Stubbe, P., and Nielsen, E.: Detection of ELF radiation from the polar electrojet (PEJ) antenna, Nature, 317, 155-157, 1985.

Barr, R. M., Stubbe, P., Rietveld, M. T., and Kopka, H.: ELF and VLF signals radiated by the "Polar Electrojet Antenna": Experimental results, J. Geophys. Res., 91, 4451-4459, 1986.

Bell, T. F. and Ngo, H. D.: Electrostatic lower hybrid waves excited by electromagnetic whistler mode waves scattering from planar magnetic-field-aligned plasma density irregularities, J. Geophys. Res., 95, 149-172, 1990.

Bell, T. F., Inan, U. S., Platino, M., Kossey, P. A., and Kennedy, E. J.: Cluster observations of lower hybrid waves excited at high altitudes by electromagnetic whistler mode signals from the HAARP facility, Geophys. Res. Lett., Vol. 31, No. 6, L06124, 2004.

Bernstein, I. B.: Waves in a plasma in a magnetic field, Phys. Rev., 109, 10-21, 1958.

Canu, P., Décréau, P. M. E., Trotignon, J. G., Rauch, J. L., Seran, H. C., Fergeau, P., Lévêque, M., Martin, Ph., Sen, F. X., Le Guirriec, E., Alleyne, H., and Yearby, K.: Identification of natural plasma emissions observed close to the plasmapause by the Cluster-Whisper relaxation sounder, Ann. Geophys., 19, 16971709, 2001.

Carpenter, D. L. and Anderson, R. R.: An ISEE/Whistler model of equatorial electron density in the magnetosphere, J. Geophys. Res., 97, 1097-1108, 1992.

Cornilleau-Wehrlin, N., Chauveau, P., Louis, S., Meyer, A., Nappa, J. M., Perraut, S., Rezeau, L., Robert, P., Roux, A., de Villedary, C., de Conchy, Y., Friel, L., Harvey, C. C., Hubert, D., Lacombe, C., Manning, R., Wouters, F., Lefeuvre, F., Parrot, M., Pincon, J. L., Poirier, B., Kofman, W., and Louarn, Ph.: The Cluster SpatioTemporal Analysis of Field Fluctuations (STAFF) Experiment, Space Scie. Rev., 79, 107-136, 1997. 
Décréau, P. M. E., Fergeau, P., Krannosels'kikh, V., Lévêque, M., Martin, P., Randriamboarison, O., Sené, F. X., Trotignon, J. G., Canu, P., Mögensen, P. B., and Investigators, W.: WHISPER, a Resonance Sounder and Wave Analyzer: Performances and Perspectives for the Cluster Mission, Space Sci. Rev., 79, 157-193, 1997.

Escoubet, C. P., Schmidt, R., and Goldstein, M. L.: Cluster - Science and Mission Overview, Space Sci. Rev., 79, 11-32, 1997.

Etcheto, J., Belmont, G., Canu, P., and Trotignon, J. G.: Active sounder experiments on GEOS and ISEE, Active Experiments in Space symposium, Alpbach, ESA SP, 195, 39-46, 1983.

Ferraro, A. J., Lee, H. S., Allshouse, R., Carrol, K., Tomko, A. A., Kelly, F. J., and Joiner, R. G.: VLF/ELF radiation from the ionosphere dynamo current system modulated by powerful HF signals, J. Atmos. Terr. Phys., 44, 1113-1122, 1982.

Gallagher, D. L., Craven, P. D., and Comfort, R. H.: Global Core Plasma Model, J. Geophys. Res., 105, 18 819-18 833, 2000.

Getmantsev, G. G., Zuikov, N. A., Kotik, D. S., Mironenko, L. F., Mityakov, N. A., Rapoport, V. O., Sazonov, Yu. A., Trakhtengerts, V. Yu., and Erdman, V. Ya.: Detection of combination frequencies as a powerful shortwave radiation interacts with an ionospheric plasma, JETP Lett., 20, 4, 229-232, 1974.

Gurnett, D. A., Huff, R. L., and Kirchner, D. L.: The Wide-Band Plasma Wave Investigation, Space Sci. Rev., 79, 195-208, 1997.

Haselgrove, J.: Ray theory and a new method for ray tracing, Rept. of Physical Society Conference on Physics of the Ionosphere, 355-364, Cambridge Univ., Cambridge, England, 1954.

Helliwell, R. A.: Coherent VLF waves in the magnetosphere, Phil. Trans., Ser. A, 280, 137-149, Roy. Soc. London, 1975.

Helliwell, R. A. and Katsufrakis, J. P.: VLF wave injection into the magnetosphere from Siple Station, Antarctica, J. Geophys. Res., 79, 2511-2518, 1974.

Inan, U. S. and Bell, T. F.: The Plasmapause as a VLF wave guide, J. Geophys. Res., 82, 2819-2827, 1977.

Inan, U. S. and Helliwell, R. A.: Active experiments from ground, Results of the Arcad 3 Project and of Recent Programmes in Magnetospheric and Ionospheric Physics Toulouse, 84, 599-607, Cepadues-Editions, Toulouse, France, 1985.

Inan, U. S., Bell, T. F., and Helliwell, R. A.: Nonlinear pitch angle scattering of energetic electrons by coherent VLF waves in the magnetosphere, J. Geophys. Res., 83, 3235-3253, 1978.

James, H. G., Dowden, R. L., Rietveld, P., Stubbe, P., and Kopka, H.: Simultaneous observations of ELF waves from an artificially modulated auroral electrojet in space and on the ground, J. Geophys. Res., 89, 1655-1666, 1984.

James, H. G., Inan, U. S., and Rietveld, M. T.: Observations on the DE 1 Spacecraft of ELF/VLF Waves Generated by an Ionospheric Heater, J. Geophys. Res., 95, 12 187-12 195, 1990.
Kapustin, I. N., Pertsovsky, R. A., Vasil'ev, A. N., Smirnov, V. S., Raspopov, O. M., Solov'eva, L. E., Ul'yachenko, A. A., Arykov, A. A., and Galakhova, N. V.: Generation of radiation at combination frequencies in the region of the auroral electrojet, JETP Lett., 25, 5, 248-251, 1977.

Kimura, I.: Effects of ions on whistler-mode ray tracing, Radio Sci.,1, 269-283, 1966.

Kotik, D. S. and Trakhtengerts, V. Yu.: Mechanism for the excitation of combination frequencies in an ionospheric plasma, JETP Lett., 21, no. 2, 114-118, 1975.

Lefeuvre, F., Rauch, J. L., Dee, V. I., Titova, E. E., and Yurov, V. E.: Detection from Aureol-3 of the modulation of auroral electrojet by HF-heating from ELF signals in the upper atmosphere above Troms $\varnothing$, Results of the Arcad 3 Project and of Recent Programmes in Magnetospheric and Ionospheric Physics Toulouse, 84, 609-619, Cepadues-Editions, Toulouse, France, 1985.

Persoon, A. M., Gurnett, D. A., and Shawhan, S. D.: Polar cap densities from DE-1 plasma wave observations, J. Geophys. Res., 88, $10123-10136,1983$.

Platino, M., Inan, U. S., and Bell, T. F.: Lightning generated whistlers observed with the Cluster satellites outside the plasmasphere, Eos Trans. AGU, 83(47), Fall Meet. Suppl., Abstract SM52A-0557, 2002.

Rietveld, M. T., Stubbe, P., and Kopka, H.: On the frequency dependence of ELF/VLF waves produced by modulated ionospheric heating, Radio Sci., 24, 270-278, 1989.

Stubbe, P. and Kopka, H.: Modulation of the polar electrojet by powerful HF waves, J. Geophys. Res., 82, 2319-2325, 1977.

Stubbe, P., Kopka, H., and Dowden, R. L.: Generation of ELF and VLF waves by polar electrojet modulation: Experimental Results, J. Geophys. Res., 86, 9073-9078, 1981.

Stubbe, P., Kopka, H., Rietveld, M. T., and Dowden, R. L.: ELF and VLF wave generation by modulated heating of the current carrying of the lower ionosphere, J. Atmos. Terr. Phys., 44, 11231125, 1982.

Trotignon, J. G., Etcheto, J., and Thouvenin, J. P.: Automatic determination of the electron density measured by the relaxation sounder on board ISEE-1, J. Geophys. Res., 91, 4302-4320, 1986.

Trotignon, J. G., Décréau, P. M. E., Rauch, J. L., Randriamboarison, O., Krasnoselskikh, V., Canu, P., Alleyne, H., Yearby, K., Le Guirriec, E., Séran, H. C., Sen, F. X., Martin, Ph., Lévêque, M., and Fergeau, P.: How to determine the thermal electron density and the magnetic field strength from the Cluster/Whisper observations around the Earth, Ann. Geophys., 19, 1711-1720, 2001.

Trotignon, J. G., Rauch, J. L., Décréau, P. M. E., Canu, P., and Lemaire, J.: Active and passive plasma wave investigation in the Earth's environment: the Cluster/WHISPER experiment, Adv. Space Res., 31, (5)1449-(5)1454, 2003.

Yabroff, I.: Computation of whistler ray paths, J. Res., NBS, 65D, 485-505, 1961. 